\title{
Transmittance enhancement at Graphene/Al interfaces
}

Haozhe Wang1, Sidan Fu2, Jifeng Liu2 and Jing Kong*1

1 Department of Electrical Engineering and Computer Science, Massachusetts Institute of Technology, 77 Massachusetts Avenue, Cambridge, Massachusetts 02139, United States

2 Thayer School of Engineering, Dartmouth College, 14 Engineering Drive, Hanover, New Hampshire 03755, United States

Email: jingkong@mit.edu

When two metal films stack together forming "hetero-film", it has been generally accepted that the effective transparency is lower than the respective metal film as a result of the absorption accumulation. Here, we report a counterintuitive phenomenon where transparency of a hetero-film is significantly higher compared to the original metal film1. Specifically, we found that by layering one-atom-thick graphene on an aluminum (Al) film, the transparency of the Al film is dramatically increased from $\sim 60 \%$ to $80 \%$ under $550 \mathrm{~nm}$ wavelength light. In this work, we investigated the optical properties of graphene/aluminum interfaces. CVD grown 1LG was firstly grown and transferred on $\mathrm{SiO}_{2}$ substrate by a PMMA-assisted approach2. Subsequently, an aluminum coating with a thickness of 4-20nm was produced by an e-beam evaporator with $99.99 \%$ purity aluminum target. We acquired the transmittance spectra of graphene/aluminum stacks using a UV-vis spectrophotometer. One interesting observation is transmittance increased in samples with graphene, indicating a novel physical or chemical interaction between graphene and aluminum. For 4nm Al film, graphene induced transparency enhancement at UV range of 200 to $300 \mathrm{~nm}$. As film thickness increases to $8 \mathrm{~nm}$, the transparency enhancement extends to a wider UV range of 10-660nm. In $12 \mathrm{~nm}$ sample, we observe an averaged $12 \%$ increase in transmittance for the wavelength range of 500-2500 $\mathrm{nm}$ in the sample with graphene, comparing with pure $\mathrm{Al}$ coating on the substrate. More surprisingly, similar transparency enhancement is captured when $\mathrm{Al}$ film was deposited on the graphene film. Due to the counter-intuitive observation, we anticipate this work will benefit the community in fundamental understanding and reliable utilization of graphene and $\mathrm{Al}$ interactions.

A
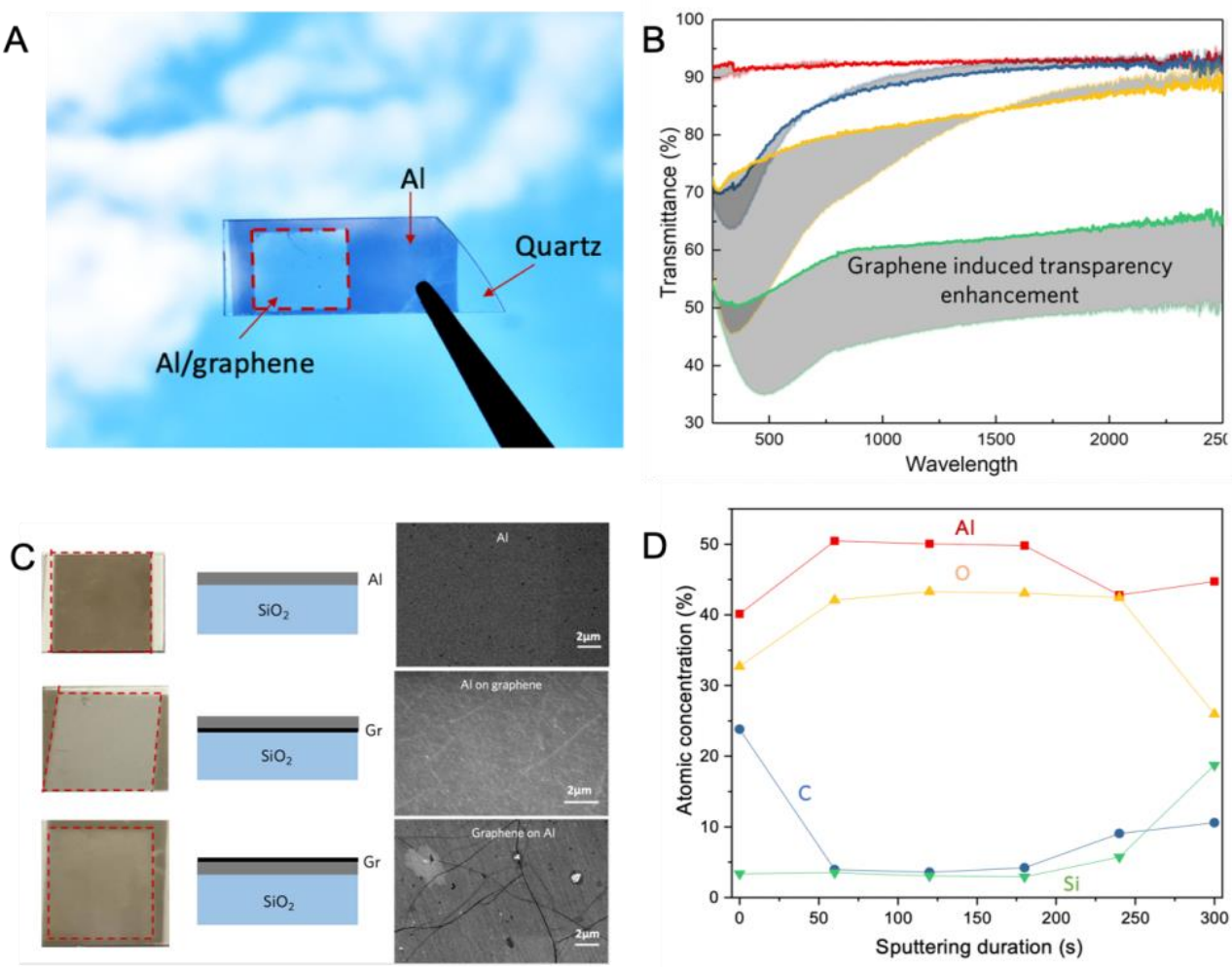

Figure 1 (A) Image to show the transmittance enhancement at Al/graphene interface; (B) Transmittance in the samples with and without graphene; (C) Surface morphologies of $\mathrm{Al} / \mathrm{SiO} 2, \mathrm{Al} / \mathrm{Gr} / \mathrm{SiO} 2$ and $\mathrm{Gr} / \mathrm{Al} / \mathrm{SiO} 2$ samples (D) XPS depth-profile of $\mathrm{Al} / \mathrm{Gr} / \mathrm{SiO} 2$ sample. 


\section{References:}

1. Wang, Haozhe, et al. "Optical Properties of Graphene/Al Hetero-film." APS Meeting Abstracts. 2019.

2. Fu, Sidan, et al. "Self-Assembled, Ultrahigh Refractive Index Pseudo-Periodic Sn Nanostructures for Broad-Band Infrared Photon Management in Single Layer Graphene." ACS Photonics 6.1 (2018): 50-58. 